\title{
Magnetic deformation of the white dwarf surface structure
}

\section{(PREPRINT)}

\author{
Christian Fendt, Potsdam, Germany \\ Astrophysikalisches Institut Potsdam \\ Dainis Dravins, Lund, Sweden \\ Lund Observatory
}

Received 2000 June 26; accepted 2000 July 12

\begin{abstract}
The influence of strong, large-scale magnetic fields on the structure and temperature distribution in white dwarf atmospheres is investigated. Magnetic fields may provide an additional component of pressure support, thus possibly inflating the atmosphere compared to the non-magnetic case. Since the magnetic forces are not isotropic, atmospheric properties may significantly deviate from spherical symmetry. In this paper the magnetohydrostatic equilibrium is calculated numerically in the radial direction for either for small deviations from different assumptions for the poloidal current distribution. We generally find indication that the scale height of the magnetic white dwarf atmosphere enlarges with magnetic field strength and/or poloidal current strength. This is in qualitative agreement with recent spectropolarimetric observations of Grw $+10^{\circ} 8247$. Quantitatively, we find for e.g. a mean surface poloidal field strength of $100 \mathrm{MG}$ and a toroidal field strength of 2-10 MG an increase of scale height by a factor of 10. This is indicating that already a small deviation from the initial force-free dipolar magnetic field may lead to observable effects. We further propose the method of finite elements for the solution of the two-dimensional magnetohydrostatic equilibrium including radiation transport in the diffusive approximation. We present and discuss preliminary solutions, again indicating on an expansion of the magnetized atmosphere.
\end{abstract}

Key words: stars: atmospheres stars: fundamental parameters stars: magnetic fields stars: white dwarfs

\section{Motivation}

Magnetic white dwarfs exhibit surface field strengths of the order of $5-500 \mathrm{MG}(0.5-50 \mathrm{kT})$ (see e.g. Chanmugam 1992, Landstreet 1992, Schmidt \& Smith 1995). These fields are globally extended fields, distributed over length scales of several stellar radii. In general, model calculations of magnetic white dwarf spectral lines reveal a dipolar structure of the magnetosphere, sometimes offset from the stellar center, and, occasionally, superimposed with higher order multipole moments.

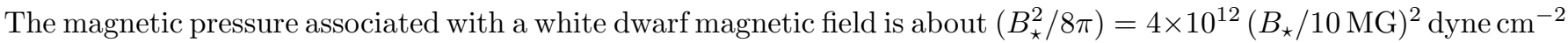
(or $4 \times 10^{11}\left(B_{\star} / \mathrm{kT}\right)^{2} \mathrm{~Pa}$ ), where $B_{\star}$ denotes the mean surface field strength. The gas pressure $P$ in the nondegenerate stellar envelope varies largely over the scale height. In the outermost regions the magnetic pressure exceeds the thermal pressure by many orders of magnitude. Hydrodynamic calculations of non-magnetic white dwarf atmospheres reveal $P \simeq 10^{6}$ dyne $^{-2}\left(10^{5} \mathrm{~Pa}\right)$ at an optical depth $\tau \simeq 1$ (Steffen et al. 1995). However, concerning the magnetohydrodynamic force-balance in the atmosphere, also magnetic tension $(\vec{B} \cdot \nabla) \vec{B}$ has to be taken into account. A dipolar magnetic field, as observed as the main field component in many white dwarfs, is essentially force-free, i.e. magnetic pressure and tension forces exactly cancel. Therefore, only non force-free field components of a white dwarf magnetic field will affect the hydrodynamic equilibrium.

The question arises, whether and how such a magnetic field will influence the global, hydrodynamic structure? And further, whether and how the radiative equilibrium and the temperature distribution is affected? If this is indeed the case, a change in the global temperature distribution along the stellar surface might be expected, eventually leading to observable effects (as solar dark spots are connected with an enhanced magnetic field strength). The most direct detection of such effects would be a periodic optical variability of a rotating single magnetic white dwarf.

A potential candidate demonstrating such effects is the very hot, highly magnetic DA white dwarf RE J0317853. Barstow et al. (1995) observe an effective temperature of $\sim 50000 \mathrm{~K}$, a field strength of $\sim 340 \mathrm{MG}(34 \mathrm{kT})$, and optical oscillations of $\sim 10 \%$ with a period of $725 \mathrm{~s}$. Another example is PG 1031+234, where the observed polarization indicates a strong magnetic spot of an offset dipolar field component (Schmidt et al. 1986). Due to the two dipole components, the magnetic field strength varies by a factor of 5 across the stellar surface. The 
observed rotational period is $3^{\mathrm{h}} 24^{\mathrm{m}}$. There is indication also from spectropolarimetric observations that a magnetic field affects the radial hydrostatic equilibrium (Östreicher et al. 1992, Friedrich et al. 1994). From the shape of absorption lines in Grw $+70^{\circ} 8247$ these authors concluded that magnetic white dwarfs may have "a more expanded photosphere" (Friedrich et al. 1994). The corresponding geometrical scale height at the optical depth $\tau \simeq 1$ for continuum absorption is increased by a factor of 300 .

\subsection{Models of the magnetic structure of white dwarfs}

The fundamental question of the white dwarf magnetic field origin and evolution has not yet been settled in the literature (see Chanmugam 1992 for a review).

The idea that magnetic fields of white dwarfs are generated or maintained by dynamo action is especially interesting for white dwarfs with variable magnetic field. For the example of GD 358, a white dwarf with only mild field strength of $1300 \mathrm{G}$ with variations of \pm 300 , Markiel et al. (1994) performed dynamo simulations, indicating that an $\alpha \omega$ dynamo is able to reproduce the 'observed' variation in field strength. Indeed, Thomas et al. (1995) concluded on theoretical grounds that dynamo generated magnetic fields could occur in many DB and DA white dwarfs.

As the magnetic flux in Ap stars is comparable to that of magnetic white dwarfs, it has been discussed that the magnetic fields in white dwarfs could just be a relict of (decaying) primordial stellar fields (Chanmugam 1992, Landstreet 1992). For example, Moss (1979b) theoretically investigated axisymmetric dipolar type magnetic fields in white dwarfs finding that the ratio of internal to surface magnetic flux increases with the ratio of rotational to magnetic energy. Further, convection may prevent a weak magnetic field (below $1 \mathrm{MG}$ ) to penetrate the stellar surface.

Most of the literature on the global stellar magnetic field evolution considers upper main sequence stars and not the white dwarfs. These papers concentrate on the field evolution and only rarely take into account hydrodynamical or thermal properties. In the following we will briefly review a few examples which are more relevant to our work.

Early models were presented by Moss (1975) and Mestel \& Moss (1977) investigating magnetic upper main sequence stars, especially the stability of the magnetic field configuration. Mestel et al. (1988) considered the interaction of magnetic fields, rotation and meridional circulation in the radiative envelope of stars. However, as their aim was the distribution of the differential rotation and the toroidal magnetic field, they prescribed the poloidal components of field and velocity. Furthermore, they treat a homogeneous sphere of an incompressible fluid.

In general, Lorentz forces seem to be able to reduce the effective gravitational potential in the upper atmosphere by $70-80 \%$ (Stepień 1978). Therefore, the whole atmosphere should expand. Stift (1977, 1978) presented a In the Stift $(1977,1978)$ model of the variable magnetic star $\beta$ CrB it's magnetic variation is explained by a "possible magnetic deformation", leading to a deviation from spherical symmetry and to precession. This interpretation, however, is was questioned by Moss (1979a) on the base of the stellar evolutionary status of that star. For magnetic Ap stars, Hubbard \& Dearborn (1982) theoretically find the interesting effect that, due to the additional magnetic pressure, the star is "blown up" by about $~ 20 \%$, forming a "magnetic balloon".

In the case of magnetic white dwarfs, Ostriker \& Hartwick (1968) concluded that the radius of the degenerate core increases with increasing magnetic energy in the interior. For a magnetic energy of 0.1 in units of gravitational energy, the core radius is 1.4 times larger compared to a non-magnetic core. In the similar direction goes the recent work by Suh \& Mathews (2000) who revise the mass-radius relation for magnetic white dwarfs.

Wendell et al. (1987) first presented quantitative results of the global magnetic field evolution of white dwarfs. They calculated the time-dependent MHD equations for purely poloidal fields neglecting non-radial motion and also any back reaction of the field on the stellar structure. In comparison with static models they find that the decay time scale increases, becoming even longer than the age of the star. Muslimov et al. (1995) extended this study by including the Hall effect,

The structure of the magnetic white dwarf atmosphere is more extreme than that of their possible progenitors, the Ap stars. As the stellar radius is reduced by a large factor and the atmospheric layer becomes thiner, huge gradients are implied in the hydrodynamic properties. In contrary, the magnetic field, if we assume it to have a roughly dipolar structure, is relatively constant throughout the atmosphere.

The general difficulty in calculating the action of Lorentz forces in stellar atmospheres is that the electric current distribution is uncertain (Landstreet 1987). In the case of magnetic CP stars observations give no evidence for the presence of toroidal fields in their line forming region (Landstreet 1987). Although there is not very much known about the distribution of toroidal fields below the surface of stars, electric currents should exist in the stellar interior in order to maintain a large-scale dipolar field. Further, Mestel \& Moss (1983) argue that a stellar magnetic field must have a mixed poloidal-toroidal structure in order to avoid dynamical instabilities. Outside of the star, in the very low density regions, an axisymmetric magnetic field should be essentially force-free. The question is, 
whether an electric current system in the stellar interior also extends throughout the stellar atmosphere. In the case of white dwarfs, where the extent of the atmosphere is only $\sim 1 \%$ of the whole star, we see no reason why the current should be excluded from the surface sheet (with 'surface' we denote that layer in the atmosphere, where the radiation originates).

Assuming that the magnetic field of Ap stars and white dwarfs is a slowly decaying fossil magnetic field, Landstreet (1987) estimated the amount of induction of electric current from that decay and, correspondingly, the strength of the induced Lorentz force. In such a picture the generation of electric current is self-consistently adopted by a physical process. However no detailed treatment of the local force-balance was made. Concerning the white dwarfs, he estimated that the decay-induced Lorentz force cannot be neglected for the hydrostatic structure of low-mass, hot white dwarfs. He argued that on order to affect the hydrostatic structure of the atmosphere, the Lorentz force should be at least equal in magnitude compared to the gravitational force.

Landstreet (1987) further claimed that due to the large Lorentz force a static equilibrium as discussed by Stepień (1978) is unlikely. He argued that the star cannot have large un-balanced forces acting in the outer atmosphere where gravity is unable to balance the Lorentz force. This would immediately lead to a rapid acceleration. The decay-induced Lorentz force is normal to the field lines and, as he proposes, tends to drive meridional motion across the field line, "probably mainly horizontally, since gas pressure will limit vertical motion". In turn, this would lead to electro-motive forcing currents opposed to those driven by decay. The matter will be accelerated until it is moving in a nearly force-free configuration.

Our viewpoint is the following. In the region where large Lorentz forces are present, the frozen-in field will also guide the plasma motion (see Sect.2.2). Therefore, acceleration in meridional direction across the field is suppressed by the field. The plasma will move slowly until a new non force-free magnetohydrostatic equilibrium is reached. On the long term evolution magnetic diffusion will nevertheless occur.

So far, no attempt has been undertaken to calculate the magnetohydrostatic equilibrium in the magnetic white dwarf atmosphere. Motivated by recent observations of spectropolarimetry, we thought that this assumption would be a reasonable approach to investigate the influence of the strong white dwarf magnetic field on their surface structure.

\subsection{Aim of the present study}

As the previous literature survey has shown, a fully self-consistent treatment of the magnetohydrodynamical equations is not yet feasible today. In particular, the combined problem of field generation and field evolution is far from being solved.

In contrast to the literature on the long term evolution of the magnetic field in white dwarfs (e.g. Wendell et al. 1987), we therefore concentrate on a 'snapshot' during this evolution. For a fixed time, we investigate how the hydrostatic equilibrium would be affected under the presence of a magnetic field. We prescribe the magnetic field structure and search for solutions of the magnetohydrostatic equilibrium in the white dwarf atmosphere. We will show that such solutions exist.

One may argue that a prescription of some arbitrary toroidal current distribution is physically not meaningful. However, it is yet the only way to obtain some quantitative results, since self-consistent magnetohydrodynamic models of the whole star are not yet possible to calculate. We further note that we do not calculate a global stellar model, but concentrate only on the atmospheric layer of the white dwarf. A treatment of the MHD of the whole white dwarf is not yet numerically feasible. On the other hand, the field structure (poloidal and toroidal) is certainly determined by the inner structure of the star, which we treat as a kind of boundary condition for the atmosphere. Since the lack of knowledge about the true toroidal field / poloidal electric current distribution, it seems appropriate to us to discuss different examples for the current distribution in order to study how they affect the hydrostatic structure of the atmosphere.

In the present paper, we consider the case only small deviation from a force-free dipolar structure. Therefore, in order to study the potential influence of the small non force-free component, the arbitrary choice of a toroidal field seems to be feasible. For a fully self-consistent approach, the proper poloidal field distribution has to be calculated from the current distribution (see Eq.10). We concentrate on a quantitative treatment of the local equilibrium, rather than treating the star as a whole.

We assume a poloidal magnetic field structure of dipolar type, but in general allow for a small deviation, either as a poloidal non-dipolar component or an additional toroidal field. Strong toroidal fields in white dwarfs are not implausible if generated by differential rotation (Muslimov et al. 1995). It is not known whether toroidal field penetrates white dwarf surface or remains contained in the interior. So far, only poloidal magnetic fields were derived from the observations. In the case of Ap stars the existence of large toroidal field on the surface seems to be ruled out (see Landstreet 1987). 
In summary, our basic assumption is that a distortion of the main (force-free) dipolar field will induce a Lorentz force re-arranging the hydrostatic equilibrium until a new steady state is reached. In this sense we follow the approach of Hubbard \& Dearborn (1982) and Stepień (1978). Meridional motion as possibly induced by toroidal fields (see Mestel et al. 1988), is neglected in our study.

In Sect. 2 we recall the equations of magnetohydrostatic equilibrium and discuss our basic assumptions. In Sect. 3 we investigate the magnetohydrostatic force balance in one dimension. In Sect. 4 we discuss the two dimensional problem, including radiation transfer in the diffusion limit and consider the method of finite elements. The full problem could not yet be resolved numerically, however, we will present preliminary solutions. We finally summarize our results in Sect. 5 .

We will use both cylindrical $(R, \phi, Z)$ and spherical coordinates $(r, \theta, \phi)$ depending on the symmetry of the individual problem. $(x, \phi, z)$ are cylindrical coordinates normalized to a stellar reference radius $r_{o}=10,000 \mathrm{~km}$. We will use Gaussian (and astronomical) units in general, but add SI units in parentheses whenever number values are derived.

\section{Radiative hydromagnetic equilibrium}

\subsection{Basic assumptions, notation}

Throughout the paper we will apply the following assumptions which simplify the general problem significantly. (i) A static equilibrium, (ii) ideal MHD (high conductivity), (iii) axisymmetry, and (iv) a grey atmosphere. Further, (v) we will prescribe the magnetic field and electric current distribution.

Real stellar atmospheres are neither static nor axisymmetric. Ongoing convection leads to non-axisymmetric structures at least on the medium spatial scale. Steffen et al. (1995) show that hydrodynamic turbulence with convection velocities of about $10 \mathrm{~km} / \mathrm{s}$ is present in the upper layers of DA white dwarf atmospheres. However, one may hypothesize that a strong magnetic field is able to suppress the turbulent motion. Moss (1979b) argues that in the case of main sequence stellar models the magnetic field structure, which depends on parameters of field energy and rotational energy, the main features follow already from radiative equilibrium models without meridional circulation. Wendell et al (1987) claim that in cooler white dwarfs the kinetic energy density in convection zones is too small in order to affect magnetic fields of strengths larger than 0.1 MG significantly. There are many white dwarfs showing evidence for stellar oscillations, but no example among the magnetic white dwarfs.

The field structure itself, derived from white dwarf spectra show that the dominant field component is in general of dipolar structure, but may also contain non-axisymmetric components leading to a total field distribution offset from the stellar center (e.g. Putney \& Jordan 1995). The field structure in the stellar interior is generally un-known. We assume that the dipolar field which is measured on the surface is a global field and just continues throughout the atmospheric layer. This is clearly questionable in respect to turbulent gas motions and the high gas pressure in the deeper layers of the atmosphere.

We neglect any effects of the magnetic field on radiation transfer and assume a grey absorption coefficient in the form of Kramer's opacity. The grey absorption assumption for white dwarf atmospheres is commonly used also for small optical depths (see e.g. Litchfield \& King 1990). It is known from both observed spectra and also spectral modeling that magnetic fields may alter the opacity (see Friedrich et al. 1994). Since we are interested in the global structure of the white dwarf atmosphere, we consider such processes as less important.

The prescription of the magnetic field is equivalent to the assumption of a stationary global field structure. Even though the field pressure widely exceeds the gas pressure in the outermost layers of the atmosphere, in the deeper layers the contrary is true. This region is applied as a boundary condition for the magnetic atmosphere.

\subsection{The assumption of ideal MHD}

In order to justify the assumption of ideal MHD for the local force-balance, we compare the time scale for magnetic diffusion, $\tau_{\text {diff }}=\left(4 \pi / c^{2}\right) \sigma_{c} R^{2}$ with the dynamical (Alfvén) time scale, $\tau_{\text {dyn }}=\left(R / B_{\star}\right) \sqrt{4 \pi \rho}$. The (Spitzer) conductivity is about $\sigma_{c} \simeq 10^{6} T^{3 / 2}$ (see e.g. Wedell et al. 1987), where $T$ is the gas temperature. A first order estimate of temperature and density can be obtained if we apply a standard white dwarf model with a grey atmosphere and a polytropic gas law (e.g. Shapiro \& Teukolsky 1983). In this case, $T=T_{\star}\left(\rho / \rho_{\star}\right)^{\gamma-1}$, where $T_{\star}$ and $\rho_{\star}$ are the critical values at the "bottom" of the atmosphere where degeneracy sets in. Choosing $T_{\star}=10^{6}$ and $\rho_{\star} \lesssim 10^{-3}$ (Shapiro \& Teukolski 1983), we derive

$$
\frac{\tau_{\text {diff }}}{\tau_{\text {dyn }}} \simeq 10^{11}\left(\frac{T}{T_{\star}}\right)^{\frac{3}{4}}\left(\frac{T_{\star}}{10^{6} \mathrm{~K}}\right)^{\frac{3}{2}}\left(\frac{\rho_{\star}}{10^{-3} \mathrm{~g} \mathrm{~cm}^{-3}}\right)^{-\frac{1}{2}}\left(\frac{R_{\star}}{10^{9} \mathrm{~cm}}\right)\left(\frac{B_{\star}}{10^{8} \mathrm{G}}\right) .
$$


This indicates that magnetic diffusion can indeed be neglected for the local force-balance, especially for hot white dwarfs. However, as it was shown in the literature (e.g. Landstreet 1987, Moss 1984) they must not be neglected for the long time magnetic field evolution of the star.

\subsection{Description of magnetic field}

Under the assumptions of stationarity and axisymmetry a magnetic flux function $\Psi(R, Z)$ can be defined,

$$
\Psi=\frac{1}{2 \pi} \int \vec{B}_{\mathrm{P}} \cdot d \vec{A}, \quad R \vec{B}_{\mathrm{P}}=\nabla \Psi \times \vec{e}_{\phi} .
$$

In the magnetostatic approach, the poloidal electric current flows parallel to the poloidal magnetic field,

$$
I=I(\Psi)=\int \vec{j}_{\mathrm{P}} \cdot d \vec{A}=\frac{c}{2} R B_{\phi} .
$$

This is similar to the case of non-static but stationary, force-free MHD.

\subsection{Radiation transfer}

The radiative equilibrium is described in the diffusive approximation,

$$
\nabla \cdot \frac{1}{\kappa \rho} \nabla T^{4}=\text { source term },
$$

with a temperature $T$, and a mass density $\rho$. This assumption is appropriate for optical depths $\tau>1$. We assume a 'grey' absorption coefficient in the form of Kramer's opacity,

$$
\kappa(\rho, T)=\kappa_{0} \rho T^{-3.5}=\kappa_{0} \frac{\mu}{\Re} P T^{-4.5}
$$

where $\kappa_{0}=4.34 \times 10^{22} \mathrm{~m}^{2} \mathrm{~kg}^{-1}$, and $\Re$ is the gas constant, $\mu$ the molecular weight of the gas particles, and $P$ the gas pressure. With Eq. (5) and the perfect gas law, $P=\Re \rho T / \mu$, the diffusion equation (4) can be rewritten in terms of $P$ and $T$,

$$
\frac{1}{\kappa_{0}} \frac{4}{9.5}\left(\frac{\Re}{\mu}\right)^{2} \nabla \cdot \frac{1}{P^{2}} \nabla T^{9.5}=\text { source term. }
$$

\subsection{Magnetohydrostatic equilibrium}

The static, hydromagnetic force equilibrium is described by

$$
\frac{1}{\rho} \nabla\left(P+P_{\mathrm{rad}}\right)=\frac{1}{4 \pi \rho}(\nabla \times \vec{B}) \times \vec{B}-\nabla \Phi .
$$

$P_{\text {rad }}$ denotes the radiation pressure. Rotational effects are negligible,

$$
\frac{\Omega^{2} R}{G M_{\star} / R^{2}} \simeq 3 \times 10^{-6}\left(\frac{P_{\star}}{1^{\mathrm{h}}}\right)^{-2}\left(\frac{R}{r_{o}}\right)^{3}\left(\frac{M_{\star}}{M_{\odot}}\right)^{-1},
$$

for a stellar rotational period $P_{\star}$ of the order of hours ( $\Omega$ is the rotational frequency). This is in contrast to e.g. Ap stars with much more extended atmospheres, where the stellar rotation becomes important. Thus, since it is the rotation that leads to meridional circulation flows, we can also neglect poloidal velocities.

The magnetic term in Eq. (7) can be rewritten in terms of the flux function $\Psi$ and the poloidal current $I(\Psi)$,

$$
(\nabla \times \vec{B}) \times \vec{B}=-\nabla \Psi\left(\nabla \cdot \frac{1}{R^{2}} \nabla \Psi+\frac{2}{c^{2} R^{2}}\left(I(\Psi)^{2}\right)^{\prime}\right),
$$

where the prime denotes the derivative $d / d \Psi$. In axisymmetry, generally

$$
R \nabla \cdot \frac{1}{R^{2}} \nabla \Psi=\frac{4 \pi}{c} j_{\phi},
$$

and the magnetic term can also be understood in terms of toroidal and poloidal currents. Equation (9) evidently shows that the magnetic field affects the force-balance only across the field (i.e. anti-parallel to $\nabla \Psi$ ). Depending 


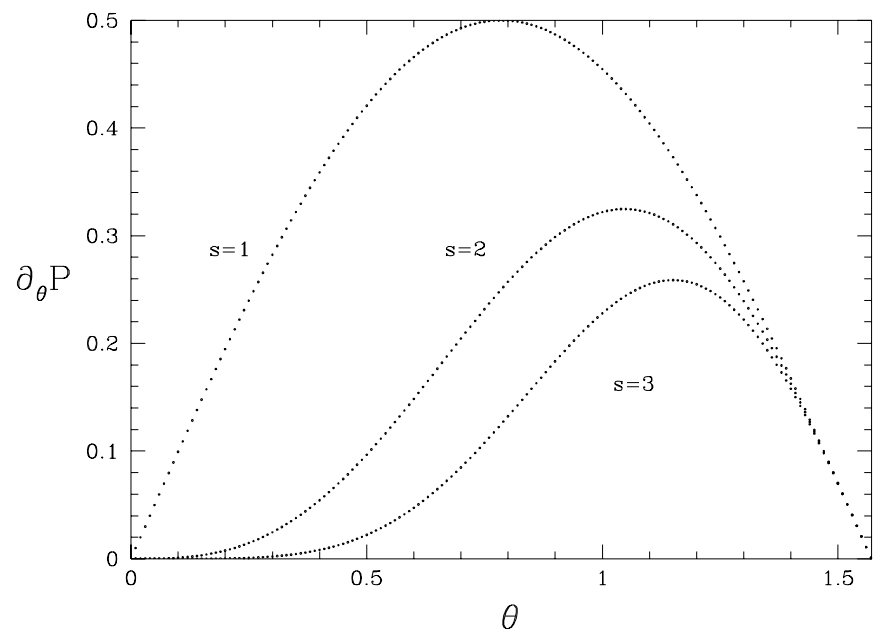

Fig. 1: Normalized gas pressure gradient in $\theta$-direction, Eq. (17), for different steepness $s$ of a model poloidal current distribution $I(\Psi) \sim \Psi^{s}$

on the field geometry, this means that the strength of the $r$ and $\theta$-derivatives varies largely with stellar latitude. In a dipolar-like distribution the $r$-derivative are small near the pole and large near the equator.

As can be seen immediately from Eq. (9), and as it was pointed out by many authors before (e.g. Stepień 1978), force-free field configurations do not contribute to the pressure equilibrium. In this case,

$$
\nabla \cdot \frac{1}{R^{2}} \nabla \Psi=-\frac{1}{R^{2}} \frac{4}{c^{2}} I(\Psi) I^{\prime}(\Psi),
$$

and the magnetic terms vanish together. In particular, for a pure dipolar field, $I(\Psi) \equiv 0$. Thus, only poloidal field geometries deviating from a dipolar structure (or, more general, from any superposition of force-free multi poles), or a toroidal field, may influence the hydrostatic equilibrium. With the perfect gas law, Eq. (9), and Poisson's equation

$$
\nabla \cdot \nabla \Phi=4 \pi G \rho
$$

we calculate the divergence of Eq. (7),

$$
\nabla \cdot T \nabla(\ln P)=-4 \pi G\left(\frac{\mu}{\Re}\right)^{2} \frac{P}{T}-\frac{4 \Re T}{\mu} \frac{P_{\mathrm{rad}}}{P}\left(\frac{\nabla T \nabla P}{T P}-4.5\left(\frac{|\nabla T|}{T}\right)^{2}\right)-\nabla \cdot\left(\frac{T \nabla \Psi}{4 \pi P}\left(\nabla \cdot \frac{1}{R^{2}} \nabla \Psi+\frac{4 I I^{\prime}}{c^{2} R^{2}}\right)\right)
$$

The factor 4.5 results from the power index in Kramer's opacity. Except for small optical depths, radiation pressure is negligible.

\subsection{Radiative magnetohydrostatic equilibrium}

With a prescribed magnetic field structure $\Psi(R, Z)$ and poloidal current distribution $I(\Psi) \sim R B_{\phi}$, Eqs. (6) and (14) represent a pair of coupled partial differential equations of second order with the unknown functions $P(R, Z)$ and $T(R, Z)$. Since the magnetic field may change the size of the stellar atmosphere, the size of the integration domain has to be adapted during the numerical computations. The stellar surface is properly determined by the condition

$$
\left|\frac{4}{3} \frac{\sigma}{\kappa \rho} \nabla T^{4}\right|=\sigma T^{4} .
$$

With that, a self-consistent solution can be obtained in an iterative process.

\section{Magnetohydrodynamic equilibrium in one dimension}

\subsection{Surface temperature distribution}

As a preliminary estimate, we calculate the global change of temperature on a sphere with constant radius $r$. In a dipolar field, the field strength varies from pole to equator by a factor of 2 and thus the magnetic pressure $P_{\text {mag }}$ by a factor of 4 , respectively. We now introduce a factor beta measuring the strength of the non force-free magnetic 

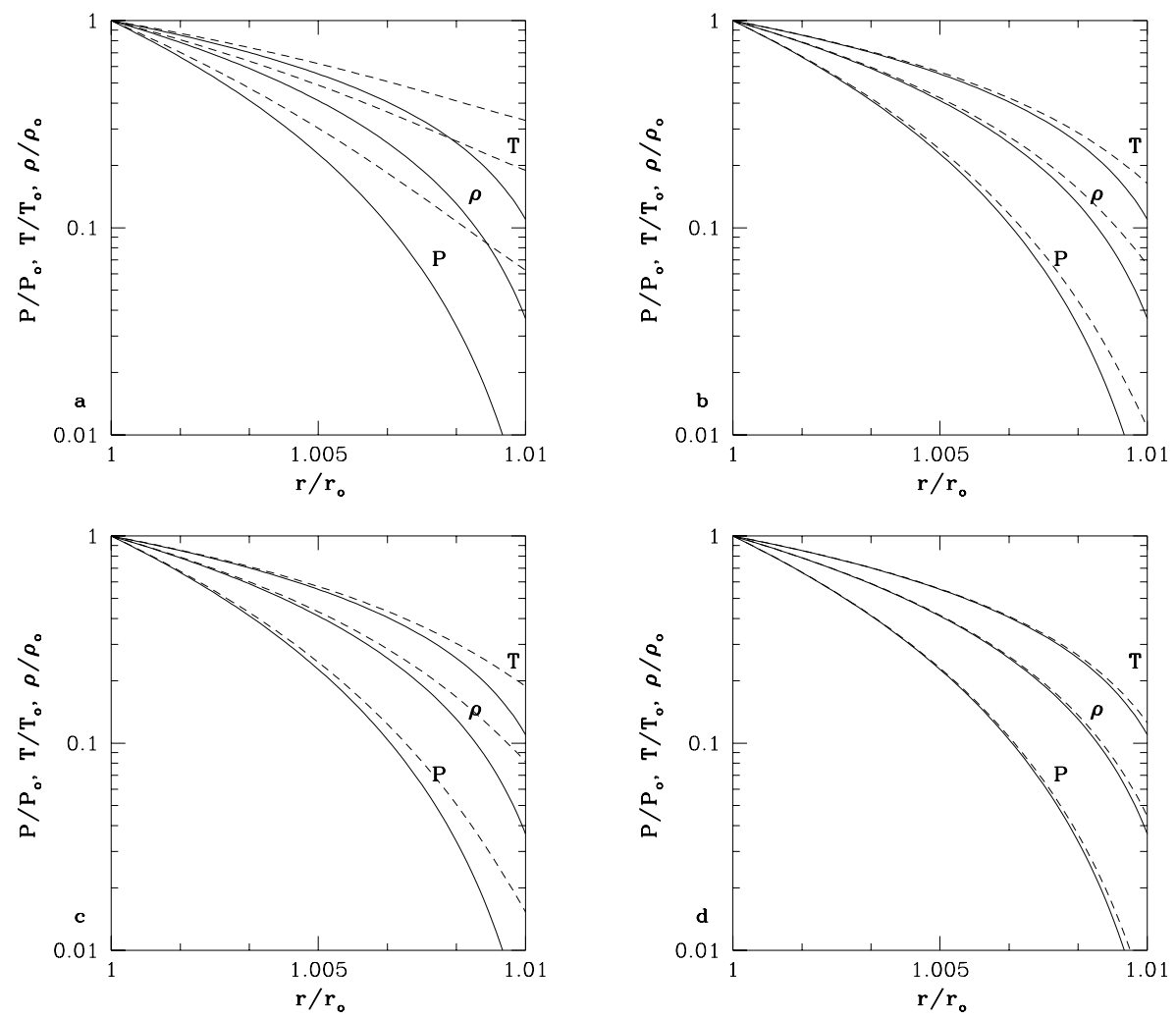

Fig. 2: Solution of the magnetohydrostatic equilibrium ignoring $\theta$-derivatives, and assuming a polytropic gas law. Gas pressure $P$, mass density $\rho$, temperature $T$, normalized to their values $P_{o}=10^{14}$ dyne $\mathrm{cm}^{-2}\left(10^{13} \mathrm{~Pa}\right), T_{o}=10^{7} \mathrm{~K}$ at the start point of the integration $r_{o}$. The polytropic index is $\gamma=5 / 3$. Solid lines denote the solution without magnetic field, dashed lines the solution under influence of magnetic fields, respectively. The latitude is $\theta=0$ (a,b), and $\theta=(\pi / 2)(\mathbf{c}, \mathbf{d})$. Field strength at $r_{o}$ is $100 \mathrm{MG}(10 \mathrm{kT})(\mathbf{a}, \mathbf{c})$, and $40 \mathrm{MG}(4 \mathrm{kT})(\mathbf{b}, \mathbf{d})$.

field component (see below). This factor should be rather low since the main field component is still the dipolar field. With the assumption that the total pressure (gas pressure plus magnetic pressure) is only a function of spherical radius, $P_{\mathrm{tot}}(r, \theta, \phi)=P_{\mathrm{tot}}(r)$, the change of gas pressure by the magnetic pressure can be estimated. For a polytropic gas law, $P \sim \rho^{\gamma}, T \sim P^{(\gamma-1) / \gamma}$, the ratio between the equatorial and the polar temperature at a certain radius is

$$
\frac{T_{\mathrm{eq}}}{T_{\mathrm{pole}}}=\left(\frac{1-P_{\mathrm{eq}} / P_{\mathrm{tot}}}{1-P_{\mathrm{pole}} / P_{\mathrm{tot}}}\right)^{\frac{\gamma-1}{\gamma}}=\left(\frac{1-(4 / 9) \beta}{1-(16 / 9) \beta}\right)^{\frac{\gamma-1}{\gamma}}
$$

We define $\beta \equiv B_{\star}^{2} /\left(8 \pi P_{\text {tot }}\right)$, where $B_{\star}=\left(B_{\text {eq }}+B_{\text {pole }}\right) / 2$. We interpret $\beta$ as an "effectiveness factor" concerning the influence of the magnetic pressure on the thermal pressure distribution, having the following argumentation in mind. Yet, magnetic tension, $(\vec{B} \cdot \nabla) \vec{B}$, was not considered in our estimate. For a dipolar field, magnetic tension exactly cancels the forces exerted by the magnetic pressure gradient (a pure dipolar field is force-free). The effectiveness factor $\beta$ can be understood as a measure of the non force-free component of the global field. These may be poloidal currents (or, equivalently, toroidal fields), or a non force-free component of the poloidal field. In Eq. (15) it is implicitly assumed that the non force-free components depend linearly on the field pressure of the axisymmetric component.

With $\gamma=(5 / 3)$ and choosing $\beta=0.1$ the temperature variation due to the global (non force-free) field is about $6 \%$. With $\beta=0.3$ the temperature variation increases to $20 \%$. Since the luminosity scales with $T^{4}$, the contribution from different stellar latitudes might vary remarkably, even for small $\beta$. $\beta=0.1$ then refers to $13 \%$ change in luminosity between polar and equatorial surface elements. In this picture the magnetic poles might be considered as 'dark spots' on the stellar surface. The true temperature distribution depends on the distribution of the electric current and non force-free field components.

From Eq. (7), it can be seen directly that a magnetic field, which is not spherically symmetric, also implies an asymmetry in the gas pressure distribution. If Eq. (7) is re-written in spherical coordinates with a gravitational 
potential $\Phi(r)=-(G M / r)$ and neglecting the radiation pressure, the $\theta$-derivative of the pressure $\partial_{\theta} P$ is

$$
\partial_{\theta} P(r, \theta)=-\frac{1}{4 \pi} \frac{\partial_{\theta} \Psi}{r^{2} \sin ^{2} \theta} \frac{4}{c^{2}} I(\Psi) I^{\prime}(\Psi) .
$$

For e.g. a dipolar field structure $\left(\nabla \cdot\left(1 / R^{2}\right) \nabla \Psi=0\right)$, and a power law poloidal current distribution $I(\Psi)=I_{\max } \Psi^{s}$, it simply follows

$$
\partial_{\theta} P(r, \theta) \sim \frac{1}{r^{2 s+2}}(\sin \theta)^{4 s-3} \cos \theta,
$$

which is shown in Fig. 1. The non spherically symmetric gas pressure distribution as a result of poloidal currents is evident. In the case of dipolar fields and with a linear current distribution, this effect scales with $r^{-4}$ and will influence different layers with different strength.

\subsection{An estimate of a critical radius for the expected magnetic effects}

In the 1D limit, the differential equation for hydrostatic equilibrium can be integrated analytically, applying diffusive radiation transfer, a perfect gas law, and Kramer's opacity approximation (see e.g. Shapiro \& Teukolsky 1983, Hansen \& Kawaler 1994), revealing $P(r) \sim T^{4.25}(r)$. This approach of a grey atmosphere corresponds to a polytropic gas law with $\gamma=(17 / 13)$. The approximation $P\left(R_{\star}\right)=0=T\left(R_{\star}\right)$ gives

$$
T(r)=7.55 \times 10^{8} \mathrm{~K}\left(\frac{R_{\star}}{r}-1\right)\left(\frac{M_{\star}}{M_{\odot}}\right)\left(\frac{R_{\star}}{r_{o}}\right)^{-1} .
$$

With Eq. (18) and $P(r) \sim T^{4.25}(r)$, a "critical temperature" can be derived, where magnetic effects may become important, $P_{\mathrm{mag}} \simeq P$. The critical temperature is of the order of

$$
T_{\text {crit }}=3.6 \times 10^{5} \mathrm{~K}\left(\frac{B_{\star}}{500 \mathrm{~T}}\right)^{4 / 17}\left(\frac{M}{M_{\odot}}\right)^{-2 / 17}\left(\frac{L \mu}{10^{-3} L_{\odot}}\right)^{2 / 17} .
$$

This corresponds to a critical optical depth in a grey atmosphere (with $\kappa$ as in Eq. 5),

$$
\tau_{\text {crit }} \simeq 1.2 \times 10^{6}\left(\frac{T_{\text {eff }}}{10^{4} \mathrm{~K}}\right)^{-4},
$$

with otherwise the same parameters as in Eq. (19), and a geometrical depth of $\simeq 10^{-4} R_{\star}$.

The main conclusion from this estimate is that the magnetic field potentially may affect the hydrostatic structure in the atmosphere over a substantial region. Clearly, the choice of $500 \mathrm{~T}$ for the comparison with the gas pressure overestimates the effect, since only the non force-free field component may dynamically influence the matter. Note that this rough estimate gives a result completely different from the consideration of Jordan (1992) who calculated the Lorentz force from electric currents rising in a conductive atmosphere. In our estimate, the one-dimensional limit is reasonable, since the white dwarf atmosphere is very thin compared to the extension of the field, the field variation being about $0.3 \%$ along a radial scale of $0.001 R_{\star}$.

\subsection{Integration of the radial magnetohydrostatic equilibrium}

Here we integrate the radial component of the simplified magnetohydrostatic equilibrium, $\nabla P=-\rho \nabla \Phi-\nabla P_{\mathrm{mag}}$, in spherical coordinates. The underlying assumption is that the $\theta$-derivatives are small compared to the $r$-derivatives. If we assume a dipolar-like distribution of the poloidal field and a vanishing toroidal field, the magnetohydrostatic equilibrium is described by

$$
\frac{d P}{d r}=-\frac{G M \rho_{o}}{r_{o} P_{o}} \frac{P^{1 / \gamma}}{r^{2}}+\frac{B_{o}^{2} / 8 \pi}{P_{o}}\left(1-\frac{3}{4} \sin (\theta)^{2}\right) \frac{24}{r^{7}}
$$

where $r, P, B^{2}$, are normalized to their values at the radius of the lower boundary of the non-degenerate atmosphere $r_{o}$ (note that here the radius $r_{o}$ is defined differently to the stellar radius $R_{\star}$ in Sect. 3.2). $T_{o}=(\Re / \mu)\left(P_{o} / \rho_{o}\right)$ is the temperature at this point. The parameters of the stellar core (luminosity, mass, radius) are the same for all solutions presented in this and the following subsection. The strength of the r.h.s. terms of Eq. (21) scales with the ratio of potential energy density to gas pressure at the lower surface, and magnetic pressure to gas pressure, respectively. 

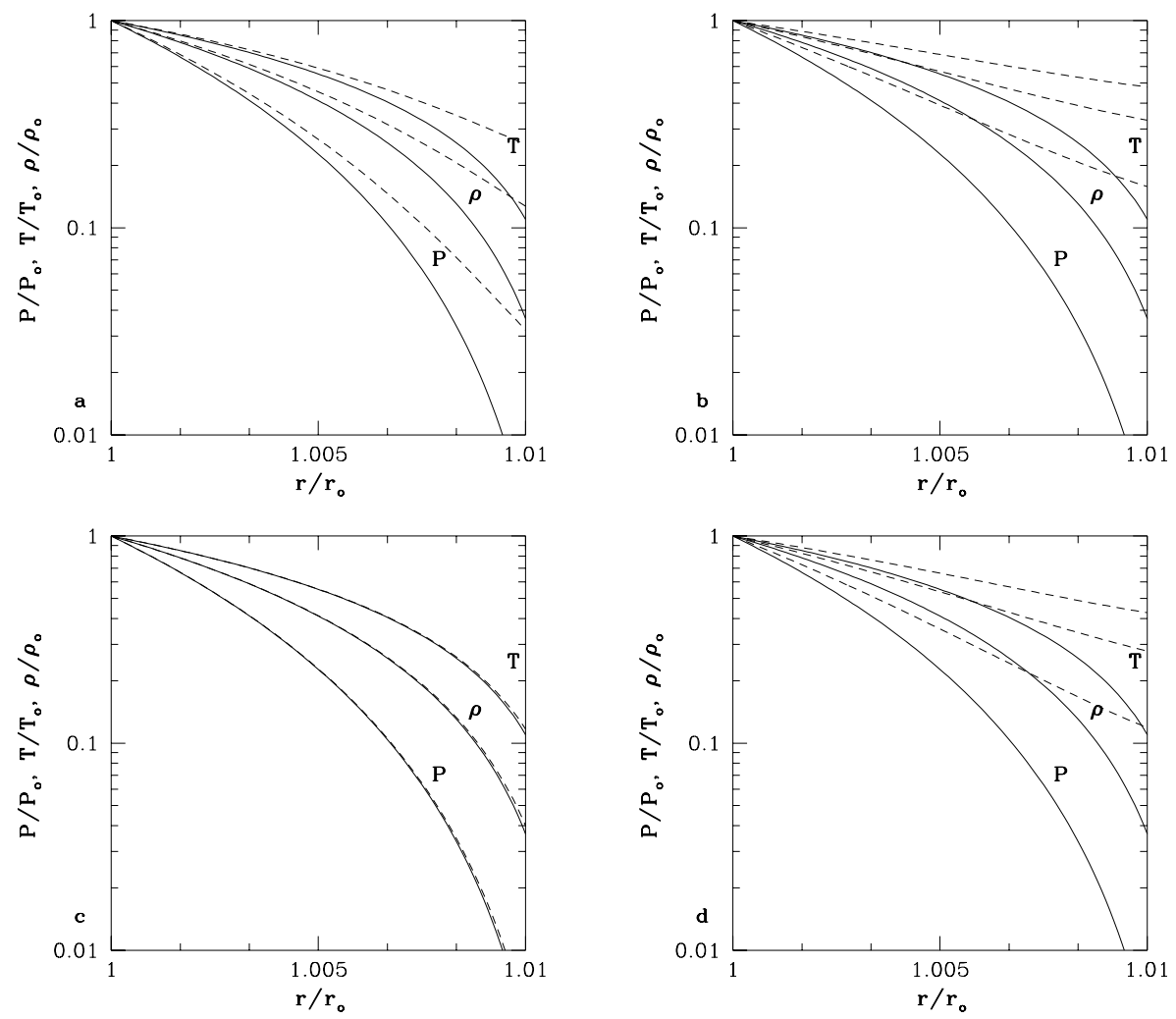

Fig. 3: Solution of the magnetohydrostatic equilibrium including toroidal fields / poloidal currents and assuming a polytropic gas law $(\gamma=5 / 3)$. $\theta$-derivatives are neglected. The latitude is $\theta=(\pi / 2) . P$, mass density $\rho$, temperature $T$, normalized to their values $P_{o}=10^{14} \mathrm{dyne}^{-2}\left(10^{13} \mathrm{~Pa}\right), T_{o}=10^{7} \mathrm{~K}$ at the start point of the integration $r_{o}$. Poloidal field strength at $r_{o}$ is $B_{o}=100 \mathrm{MG}(10 \mathrm{kT})$. Poloidal current distribution $I(\Psi)=A \Psi^{n}$. Solid lines denote the solution without magnetic field, dashed lines the solution under influence of magnetic fields, respectively. Parameters: a $\beta_{c}=0.01, n=0.1, I_{\max }=510^{12} \mathrm{~A}, \mathbf{b} \beta_{c}=0.02, n=0.1, I_{\max }=110^{13} \mathrm{~A}, \mathbf{c} \beta_{c}=0.01, n=0.2, I_{\max }=510^{12} \mathrm{~A}, \mathbf{d}$ $\beta_{c}=0.1, n=0.2, I_{\max }=510^{14} \mathrm{~A}$

Figure 2 shows solutions of Eq. (21) for different field strength $B_{o}$, and for different stellar latitudes $\theta$ (We have used the mathematical software package Mathcad for integrating Eq. (21)). The general result is that the magnetic pressure gradient leads to an increase of scale height of the atmosphere. The gas pressure (and density and temperature) decreases faster in the case without any magnetic field gradient. This effect is larger near the axis, since the magnetic term in Eq. (21) is strongest for $\theta=0$, although the $r$-gradients are larger near the equator. At the height of about $0.01 r_{o}$ the gas pressure under influence of magnetic field gradients is 15 times larger than without magnetic effects. For the gas density this factor is 6 (see Fig. 2a).

In the case of very strong fields one observes an inversion of the density profile at a certain radius, which is unphysical and shows the limits of the current investigation. The results presented in Fig. 2 remain valid for any other global field structure, for which the magnetic term is equivalent to the one in Eq. (21). In such a case, $B_{o}$ can be understood as the non-dipolar component of the dipolar-like structure, similar to Sect.3.1., and $B_{o}=\beta B_{\star}$. The numerical results will scale equivalently.

\subsection{Integration of the radial magnetohydrostatic equilibrium including poloidal currents}

We now consider poloidal currents in the force-balance. We assume a global dipolar poloidal field (thus, the first part in the parenthesis in Eq. (9) vanishes), and prescribe, artificially, a poloidal current along the field lines $I(\Psi)$. Since the static magnetic forces direct across the field (see Eq. (9)), they will predominantly influence the hydrostatic equilibrium at lower stellar latitudes, in difference to the results of previous section. If we assume an underlying dipolar field, $\nabla \Psi$ is anti-parallel to the radius vector.

A first constraint for a realistic current distribution is that the current must vanish along the symmetry axis, $I(\Psi=0)$. Therefore, in an axisymmetric magnetic field with dipolar geometry Lorentz forces have no effect on the hydrostatic equilibrium along the symmetry axis. The force-balance along the rotational axis is purely hydrostatic. This is contrary to the scenario in Sect. 3.1. and 3.3., where we assumed that the magnetic effect on the hydrostatic force-balance depends solely on the field strength. 

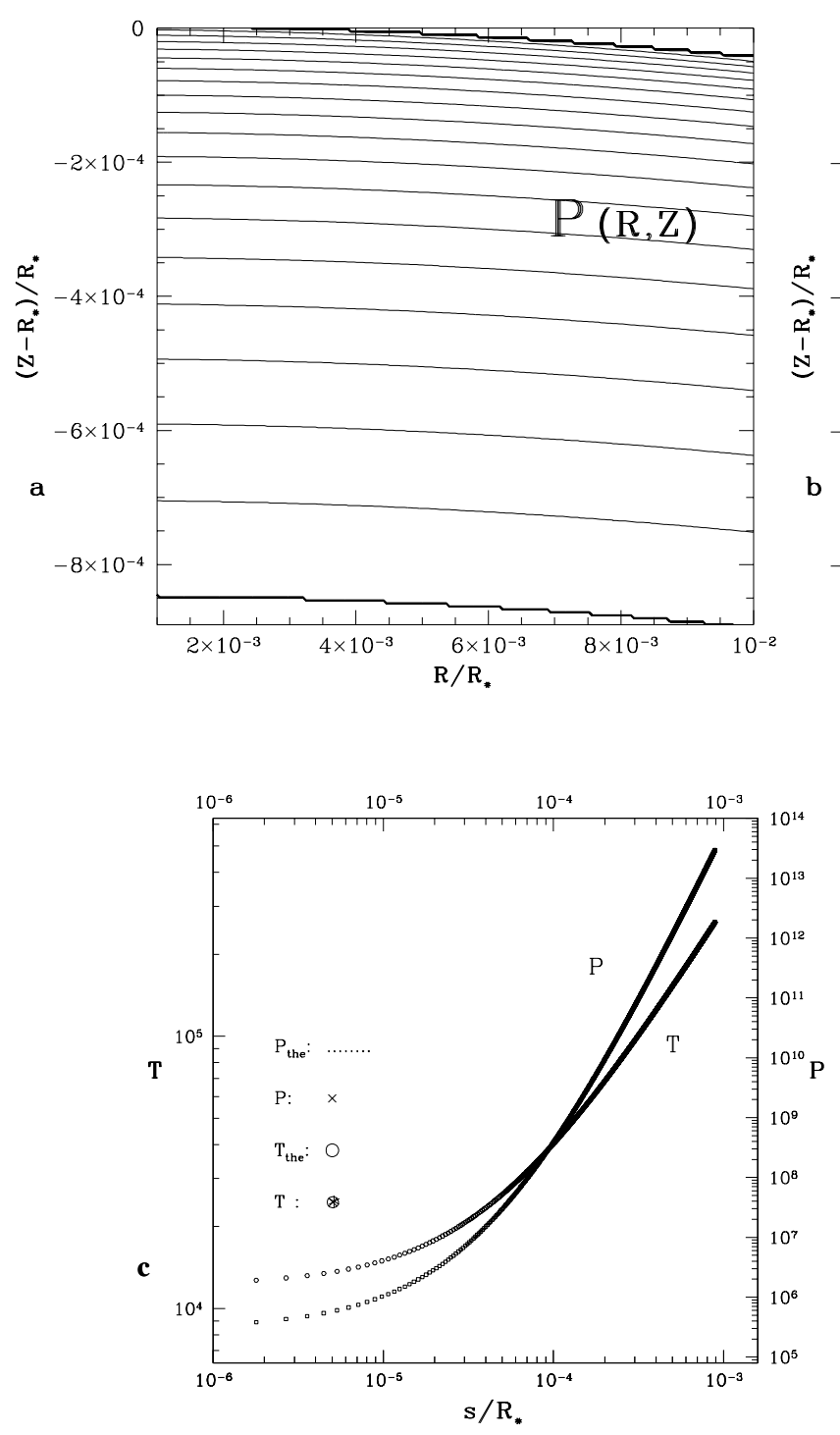

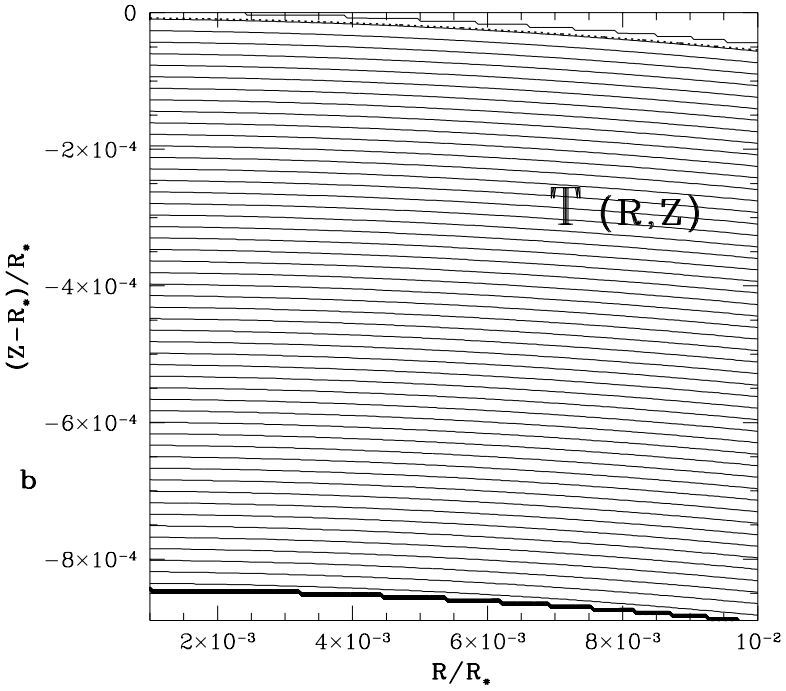

Fig. 4: Magnetohydrostatic equilibrium in the case of a non radiative atmosphere with a force-free dipolar magnetic field of $10 \mathrm{MG}$ $(1 \mathrm{kT})$ field strength. Isocontours of the two-dimensional a pressure and $\mathbf{b}$ temperature distribution. Contour levels: $P=$ $\exp (n)$ dyne $\mathrm{cm}^{-2}$ (0.1 Pa), $n=5,30,1 ; T=$ $n K, n=10000,300000,5000$. c Pressure and temperature profile, $P$ and $T$, along a radius vector near the right boundary in Figs. $4 \mathrm{a}$,b. $s$ is the normalized geometrical depth. (Note that there are four curves, representing the theoretical (labeled with "the") and the numerical distribution, respectively)

We chose the model poloidal current distribution as $I(\Psi)=A \Psi^{n}$. The parameter $A$ is related to the maximum poloidal current $I_{\max }$ with $A=I_{\max }\left(1 / B_{o} r_{o}^{2}\right)^{n}$. Then, for a dipolar field the radial component of the forceequilibrium is

$$
\frac{d P}{d r}=-\frac{G M \rho_{o}}{r_{o} P_{o}} \frac{P^{1 / \gamma}}{r^{2}}-\frac{B_{o}^{2}}{8 \pi} \frac{2 n \beta_{c}^{2}}{P_{o} r_{o}^{2 n-1}} \frac{\sin (\theta)^{4 n-2}}{r^{4 n+2}} .
$$

The parameter $\beta_{c}=\left(B_{\phi} / B_{o}\right)$ is defined as the ratio between the poloidal and toroidal field strength, and $A \equiv$ $(c / 2)\left(B_{o} / r_{o}\right) \beta_{c}$. It is equivalent to the parameter $\beta$ introduced in Sect. 3.1.

Figure 3 shows the solution of Eq. (22) along the equatorial plane for different strengths of the poloidal current (again, the Mathcad package was used for the integration procedure). Similar to the results in Sect. 3.3 the atmospheric scale height increases. Again, for large currents an inversion of the density profile is indicated. The results in Fig. 3b,d, are close to this limit. 


\section{The $2 \mathrm{D}$ radiative magnetohydrostatic equilibrium}

In Sect. 2 we have formulated the equations of magnetohydrostatic equilibrium and diffusive radiation transfer as partial differential equations of second order with non-linear source terms. In order to solve this type of equations the method of finite elements (see Camenzind 1987, Fendt et al. 1995 for further discussion and tests of the code) is a suitable numerical approach. The mesh of finite elements can be easily adapted to any shape of the physical integration region. Further, the finite element approach allows for a solution basically to any choice of boundary conditions. In this section we discuss a first approach to numerical solutions of the two-dimensional magnetohydrostatic equilibrium.

Besides the general advantage in using the method of finite elements, the problem itself carries its own special difficulty. On one hand we intend to solve the boundary value problem, in particular defined by the boundary of the stellar surface. But on the other hand, we do not know the position of that surface. One of our aims is exactly to find the "proper" stellar surface under influence of magnetic fields.

In particular, $\tau<<1$ and $\tau>>1$ might be chosen as upper and lower boundaries. However, the diffusive radiation transfer assumption breaks down for small optical depths, and the solution would not be self-consistent anymore. The choice of the upper boundary to be close to the optical depth $\tau \simeq 1$ is somehow in contradiction with the motivation to calculate a "new" temperature distribution along the stellar surface, since this is where the observed radiation originates. Therefore, this region should not be too close to any prescribed boundary condition.

For this preliminary investigation we chose the following approach. Since at the lower boundary magnetic effects are negligible, we use the analytical (hydrostatic) solution as a boundary condition for the gas pressure and temperature,

$$
P^{2}=\left(\frac{2}{8.5} \frac{16 \pi a c G M_{\star}}{3 \kappa_{0} L_{\star}} \frac{\Re}{\mu}\right) T^{8.5}\left(\frac{1-\left(T_{o} / T\right)^{8.5}}{1-\left(P_{o} / P\right)^{2}}\right),
$$

(e.g. Hansen \& Kawaler 1994). With $P_{o}<<P_{l}, T_{o}<<T_{l}$ the gas pressure at the lower boundary follows directly from the choice of the lower temperature. Along the (spherically) radial boundaries we apply homogeneous Neumann boundary conditions. As the lower boundary we choose a certain geometrical depth and the temperature/pressure according to Eq. (18) and (23). Along the outer boundary we take a gas pressure of $P=310^{5}$ dyne $^{-2}\left(310^{4} \mathrm{~Pa}\right)$ adapted from the literature (Steffen et al. 1995) at $\tau=0$. The integration domain is about $10^{-3} R_{\star}$ in the radial direction and $\leq 10^{-2} R_{\star}$ in a direction tangential to the stellar surface.

We use the following parameters. A stellar mass of $M_{\star}=0.75 M_{\odot}$, a luminosity of $2.5 \times 10^{-4} L_{\odot}$, a radius $R_{\star}=10,000 \mathrm{~km}$, an effective temperature $T_{\text {eff }}=12200 \mathrm{~K}$, an effective atomic mass $\mu=1.4$.

\subsection{The test example - the numerical solution with a polytropic gas law and force-free magnetic field}

If we neglect the radiative terms in Eq. (13), this equation allows for a investigation of electromagnetic effects on the hydrostatic equilibrium solely. Further, the case of a vanishing field or that of a dipolar field may serve as a test for the numerical code. Then, a polytropic temperature law $T(P)$ instead of Eq. (6) is used for the iterative temperature update. Figure 4 shows such test solutions of our code for a white dwarf atmosphere with a polytropic index $\gamma=6 / 5$ and a purely dipolar field of $10 \mathrm{MG}(1 \mathrm{kT})$. The non-magnetic case gives the same result. As mentioned above, we prescribe homogeneous Neumann boundary conditions along the (spherically) radial boundaries. The numerical solution perfectly matches the analytical result similar to Eq. (23) and (18) (see also Fig. 4c).

\subsection{Non force-free magnetic field and diffusive radiation transport}

The full solution of the two-dimensional hydromagnetic equilibrium (Eq.(13) including toroidal magnetic fields and diffusive radiation transport (Eq.4) is shown in Fig. 5. The calculation is done for an region located at a stellar latitude of $\simeq 30^{\circ}$ The upper boundary (the stellar surface) was fixed, i.e. we did not consider Eq. (14). We choose the same upper boundary condition as for the solution shown in Fig. 4. The lower boundary, however, differs due to the treatment of diffusive radiation transport.

The resulting two-dimensional gas pressure distribution is shown in Fig. 5a for the the case of a force-free poloidal magnetic field and the case where additionally a toroidal field is taken into account. We have applied a current distribution $I(\Psi) \sim\left(\Psi_{\max }-\Psi\right)\left(\Psi-\Psi_{\min }\right)$, normalized in such a way that the maximum toroidal field is $B_{\phi} \leq 1000 B_{\mathrm{p}}$

For the solution considering the non force-free magnetic fields components, the gas pressure isocontours are shifted outwards over large parts of the integration box. The maximum increase of gas pressure is a factor of 8 . We hypothesize that, if we would have calculated the "new" position of the stellar surface considering Eq. (4), also this 

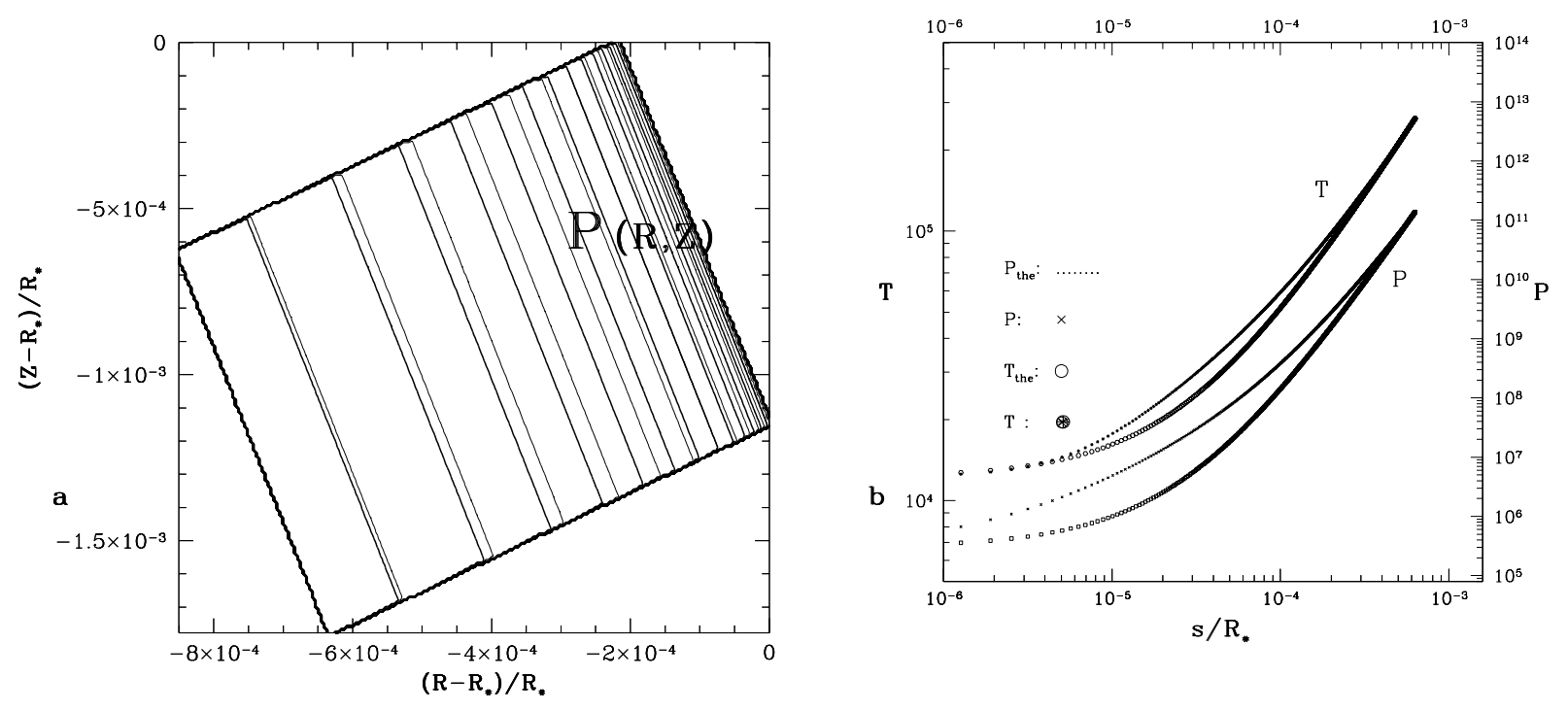

Fig. 5: Magnetohydrostatic equilibrium for a magnetized white dwarf atmosphere including diffusive radiation transport. a Isocontours of the two-dimensional pressure distribution containing a force-free field thick contours, and also a toroidal field thin contours. The integration region is located at $33^{\circ}$ of stellar latitude. The magnetic field consists of a $10 \mathrm{MG}(1 \mathrm{kT})$ dipolar field and a toroidal field (electric current) with $B_{\phi} \leq 1000 B_{\mathrm{p}}$. Contour levels: $P=\exp (n)$ dyne $\mathrm{cm}^{-2}(0.1 \mathrm{~Pa}), n=5,30,1$. $\mathbf{b}$ Distribution of pressure and temperature along a radius vector in Fig. 5a (the force-free solutions are labeled with "the").

surface would have moved outwards. We propose that the increase of radius will be different for different stellar latitudes. It is only because of our choice of the upper boundary condition that the pressure and temperature remain fixed at this boundary. In this sense the solution close to the upper boundary layer is preliminary, since the proper hydromagnetic upper boundary condition for this boundary can only be found by an iterative procedure. We defer this problem to a future paper.

In Fig. 5b we plot the (spherically) radial pressure and temperature profile close to the right boundary of the two-dimensional box in Fig. 5a. Again, for comparison, the solution in the case of a force-free magnetic field is shown. A dipolar of $10 \mathrm{MG}(1 \mathrm{kT})$ magnetic field leaves the hydrostatic equilibrium unchanged (Figs. 4c, 5b). With the additional (non force-free) toroidal field component the gas pressure and temperature increase in the intermediate region.

The relatively strong toroidal field strength is understandable, if we consider that it is the net Lorentz force of the toroidal field, i.e. the difference between the magnetic pressure gradient directed outwards, and the magnetic tension directed inwards, which changes the hydrostatic equilibrium. For a different toroidal field distribution the field strength required for the re-configuration of the hydrostatic equilibrium could be considerably lower.

The question of the observed temperature distribution of the inflated atmosphere cannot be answered yet. So far, there seem to be two possibilities for the final state of the hydromagnetic equilibrium, implying different observable effects. In one configuration the atmosphere inflates to such a structure that the gas pressure isocontours and the temperature isocontours both follow the same curve. The other possibility is that the surfaces of constant gas pressure follow a curve different from that of the temperature. This would be equivalent to a surface temperature variation along the stellar surface corresponding to a re-distribution of radiation flux in the direction perpendicular to the radius vector.

\section{Summary}

In this paper we investigated how the structure of white dwarf atmospheres is affected by a global strong magnetic field. Due to the complexity of the problem, and due to the lack of knowledge about physical properties not yet delivered by the observations, this question could only be treated under certain simplifying assumptions. Our main assumption is the prescription of the magnetic field distribution. We estimate, how the gas pressure and tenmperature changes if we add a small non force-free field component to the large-scale force-free magnetic field.

In the beginning we discussed some preliminary parameter estimates assuming that the non force-free magnetic field component is proportional to the total field strength, and its strength about $10 \%$. With that the luminosity 
of a surface element may change by $20 \%$ from polar to equatorial latitudes.

Then the magnetohydrostatic equilibrium was solved numerically in one dimension along the radius vector. We find clear evidence that the scale height of the white dwarf atmosphere increases under the additional pressure of magnetic fields. Depending on the field strength, the scale height of the atmosphere may increase by a factor of 10. This is in agreement with spectropolarimetric observations (Östreicher et al. 1992, Friedrich et al. 1994) We point out that the variation of a non force-free field component will also change the gas pressure across the surface and thus the temperature and flux distribution.

The set of equations for the axisymmetric radiative magnetohydrostatic equilibrium was re-formulated into a pair of partial differential equations of second order. These equations were solved with our code iteratively applying the method of finite elements. The two-dimensional distribution of the gas pressure and temperature in a box with a size of $0.001 \times 0.001$ stellar radii located at $33^{\circ}$ stellar latitude were presented. As a result, the gas pressure and temperature levels are shifted to higher altitudes. Although the calculations were performed for a constant size of the computational box our results gives a clear indication for an expansion of the white dwarf atmosphere under the influence of the non-force-free magnetic field. A more complete treatment would have to consider the proper determination of the true stellar surface. However, our main result, i.e. the expanding atmosphere, is not affected by that constraint. Observational indictation for an increase of the scale height in magnetic white dwarfs were found by Friedrich et al. (1994).

We hypothesize that the atmospheric expansion is different in amount for different stellar latitudes depending on the magnetic field distribution. This would lead to a non-spherically distributed gas pressure and temperature profile in the atmosphere, and, thus, to a variation of the luminosity of surface elements at different latitudes.. Such a luminosity variation could finally be observed.

Barstow et al. (1995) concluded that for the hot and highly magnetic white dwarf RE J0317-853 the most plausible explanation for the modulation of optical flux is due to the rotation of the star. A non-equal global temperature distribution along the stellar surface due to magnetoelectric effects, as discussed in the present paper, together with either an inclined rotational axis of the star or an inclined magnetic axis would explain such an intensity variation. Further observational developments, such as increased sensitivity for time-resolved spectrophotometry and polarimetry in very large telescopes, will provide a firmer experimental basis for verifying and further developing these concepts.

Acknowledgements. This work was supported by the Swedish Natural Science Research Council (NFR). C.F. thanks all members of Lund Observatory for their kind hospitality.

\section{A Numerical techniques: the finite element code}

Equations (13) and (6) are solved by means of the method of finite elements (for a more detailed discussion see Camenzind 1987, Fendt et al. 1995). For this purpose the equations are multiplied by a test function $N$ (Galerkin ansatz) and integrated over the two-dimensional plasma domain $G$ applying Green's identity. In the case of Eq. (13) we end up with

$$
\int_{G} R T \nabla N \cdot \nabla(\ln P) d R d Z=-\int_{G} J N d R d Z+\int_{\partial G} R T N \frac{\partial(\ln P)}{\partial n} d S
$$

and with a similar result for Eq. (6). $n$ denotes the unit vector perpendicular to the boundary $\partial G$, and $J(R, Z)$ is the source term on the. r.h.s. of Eq. (13) or (6).

The integration domain $G$ is discretized in a set of isoparametric curvilinear 8-node elements of the serendipity class (Schwarz 1991). Within each finite element the function $\ln P$ (or $T$ ) is expanded,

$$
\ln P(R, Z)=\sum_{i=1}^{8} \ln P_{i}^{(e)} N_{i}(\zeta, \eta)
$$

$\ln P_{i}^{(e)}$ denotes the logarithmic gas pressure at the nodal point $i$ of the element $(e)$ and $(\zeta, \eta)$ are rectilinear coordinates on the normalized element. The shape function $N_{i}$ is unity at each node $i$ and varies quadratically with $\zeta$ or $\eta$ on the edge of the (Serendipity class of) element.

Following the Galerkin scheme we select the shape functions $N_{i}$ as test function and finally obtain a system of nonlinear equations for $\ln P$ at all nodal points,

$$
\mathcal{A} \ln P=\vec{b}(\ln P),
$$

with the integrals on each grid element

$$
A_{i j}^{(e)}=\int_{G_{e}} R T\left(\partial_{x} N_{i} \partial_{x} N_{j}+\partial_{z} N_{i} \partial_{z} N_{j}\right) d x d z, \quad \text { and } \quad b_{i}^{(e)}=\int_{G_{e}} N_{i} J^{(e)} d x d z+\int_{\partial G} R T N_{i} \partial_{n}(\ln P) d s
$$


and similarly for Eq. (6) and $T(R, Z)$. Each component of Eq. (26) corresponds to the force equilibrium between neighboring nodal points of each element. Inversion of matrix equation (26) yields the solution $(\ln P$ ) for each nodal point. The expansion (25) provides the solution in any point $(\ln P(x, z))$.

\section{References}

Barstow, M.A., Jordan, S., O’Donoghue, D., Burleigh, M.R., Napiwotzki, R., Harrop-Allin, M.K., 1995: MNRAS, 277, 971 Camenzind, M., 1987: A\&A, 184, 341

Chanmugam, G., 1992: ARAA, 30, 143

Fendt Ch., Camenzind M., Appl S., 1995: A\&A, 300, 791

Friedrich S., Östreicher R., Ruder H., Zeller G., 1994, A\&A, 282, 179

Hansen, C.J., Kawaler S.D., 1994, Stellar Interiors, Springer, Berlin

Hubbard E.N., Dearborn D.S.P., 1982: ApJ, 254, 196

Jordan, S., 1992: A\&A, 265, 570

Landstreet J.D., 1987: MNRAS, 225, 437

Landstreet, J.D., 1992: RvMAMex, 5, 437

Litchfield, S.J., King, A.R., 1990: MNRAS, 247, 200

Markiel, J.A., Thomas, J.H., van Horn, H.M., 1994: ApJ, 430, 834

Mestel, L., Moss, D.L., 1977: MNRAS, 178, 27

Mestel, L., Moss, D.L., 1983: MNRAS, 204, 575

Mestel, L., Moss, D.L., Tayler, R.J., 1988: MNRAS, 231, 873

Moss, D.L., 1975: MNRAS, 173, 141

Moss, D.L., 1979a: MNRAS, 186, 185

Moss, D.L., 1979b: MNRAS, 187, 601

Moss, D.L., 1984: MNRAS, 207, 107

Muslimov, A.G., van Horn, H.M., Wood, M.A., 1995: ApJ, 442, 758

Östreicher, R., Seifert, W., Friedrich, S., Ruder, H., Schaich, M., Wolf, D., Wunner, G., 1992: A\&A, 257, 353

Ostriker, J.P., Hartwick, F.D.A., 1968: ApJ, 153, 797

Putney, A., Jordan, S., 1995: ApJ, 449, 863

Schmidt, G.D., Smith, P.S., 1995: ApJ, 448, 305

Schmidt, G.D., West, S.C., Liebert, J., Green, R.F., Stockman, H.S., 1986: ApJ, 309, 218

Schwarz, H.R., 1991: Methode der finiten Elemente, Teubner, Stuttgart

Shapiro, S.L., Teukolsky, S.A., 1983: Black Holes, White Dwarfs, and Neutron Stars, Wiley-Interscience, New York

Steffen, M., Ludwig, H.-G., Freytag, B., 1995: A\&A, 300, 473

Stepień, K., 1978: A\&A, 70, 509

Stift, M.J., 1977: MNRAS, 178, 11

Stift, M.J., 1978: MNRAS, 183, 443

Thomas, J.H., Markiel, J.A., van Horn, H.M., 1995: ApJ, 453, 403

Wendell, C.E., van Horn, H.M., Sargent, D., 1987: ApJ, 313, 284

Suh, I.-S., Mathews, G.J., 2000: ApJ, 530, 949

Addresses of the authors:

Ch. Fendt, Astrophysikalisches Institut Potsdam, An der Sternwarte 16, DE-14482 Potsdam, Germany, cfendt@aip.de D. Dravins, Lund Observatory, Box 43, SE-22100 Lund, Sweden, dainis@astro.lu.se 